\section{Isolated ARDS in a leptospirosis positive patient: An unusual sole presentation of anicteric leptospirosis.}

\author{
Keevan Singh ${ }^{1,2}$, Nickilia Maurice ${ }^{2}$ \\ ${ }^{1}$ Anaesthesia and Intensive Care Unit, Department of \\ Clinical Surgical Sciences, University of the West Indies, \\ St. Augustine, Trinidad. \\ ${ }^{2}$ Anaesthesia and Intensive Care Department, San \\ Fernando General Hospital, Trinidad.
}

\section{Corresponding Author:}

Dr. Keevan Singh

Anaesthesia and Intensive Care Department

San Fernando General Hospital, Trinidad

Email: keevansingh@gmail.com

\section{ABSTRACT}

Leptospirosis is generally a self-limiting zoonotic disease, although severe variants such as Weil's disease often warrant organ support in the Intensive Care Unit (ICU). In most endemic regions, the disease may be suspected from its associated clinical features. We present a case of a young male with a history of swimming in a freshwater lake who then presented to us with predominantly pulmonary symptoms. None of the defining clinical features of leptospirosis was present. He was intubated and ventilated for seven days in our ICU for hypoxemia and pulmonary haemorrhage. During this time, he had repeated bouts of pulmonary haemorrhage and hypoxemia while all of the organ systems typically affected by leptospirosis remained normal (platelet count, renal and liver function). Where a possible clinical exposure has occurred, leptospirosis should be considered in cases of pulmonary haemorrhage and Acute Respiratory distress syndrome (ARDS) even if classic clinical features are absent, especially in endemic regions.

\section{INTRODUCTION:}

Leptospirosis is a global zoonotic disease with protean manifestations. ${ }^{1}$ In the critical care setting, most clinicians will be familiar with the classic triad of Weil's disease which include jaundice, renal failure and haemorrhagic manifestations. ${ }^{2}$ However, the changing disease epidemiology may mean predominantly pulmonary presentations are becoming more common. ${ }^{2,3}$ Even the spectrum of pulmonary involvement can be broad, ranging from mild cough and dyspnea to severe pulmonary haemorrhagic syndrome (SPHS) and ARDS. ${ }^{2}$

In most cases, there are associated clinical features that can guide the clinician to the right diagnosis. This diagnostic challenge is increased as confirmatory testing is not routinely available in most endemic and non-endemic regions. ${ }^{4,5}$ Due to the high mortality associated with pulmonary presentations, atypical presentations can delay the diagnosis and the necessary level of care. We present a case where a young male required mechanical ventilation for pulmonary haemorrhage and ARDS. Leptospirosis was suspected from his history, however none of the other key organ specific signs were present. This case serves to highlight a rare but recognized presentation of severe leptospirosis with solely a pulmonary presentation.

\section{CASE REPORT}

A twenty-three-year-old male construction worker presented to the hospital with a five-day history of fever, headache, cough, worsening dyspnoea and generalized myalgia. He had no prior medical problems and on further questioning, gave a history of swimming in a freshwater pond approximately one week prior to the onset of his symptoms. He also had contact with rats.

On review, he had a blood pressure of $87 / 41 \mathrm{mmHg}$, a heart rate of $113 \mathrm{bpm}$ and a respiratory rate (RR) of 40 on a non-rebreathable facemask (NRBFM) delivering oxygen at $15 \mathrm{~L} / \mathrm{min}$. A chest radiograph showed bilateral diffuse patchy opacifications in both lung fields (Figure 1). Laboratory investigations at this time showed a white blood cell count (WBC) of $12.4 \times 10^{3} / \mu \mathrm{L}$, haemoglobin $(\mathrm{Hb})$ of $10.7 \mathrm{~g} / \mathrm{dL}$ and a platelet count of $170 \times 10^{3} / \mu \mathrm{L}$. Serum electrolytes were normal with a creatinine of $1.3 \mathrm{mg} / \mathrm{dL}$. 


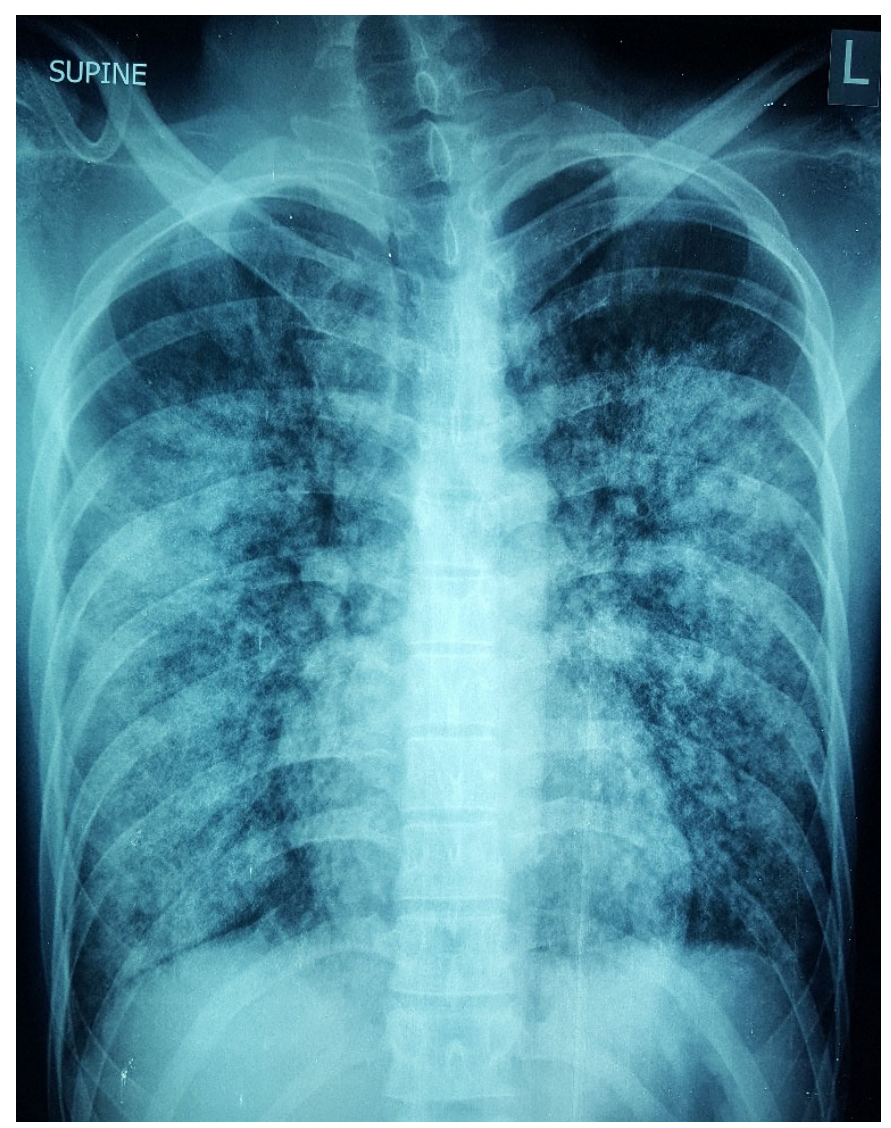

Figure 1. Patient's Chest X-ray on presentation showing bilateral alveolar opacifications and consolidations in all but the patient's upper lung fields.

A computerized tomography scan (CT) of the chest showed symmetrical diffuse alveolar opacifications with widespread background ground glass opacification. Shortly after the CT scan he deteriorated, (RR 52, oxygen saturation (SpO2) $84 \%$ on a non-rebreathable face mask) requiring intubation and mechanical ventilation. Post intubation, frank blood was noted via his endotracheal tube and he was transferred to the ICU.

In the ICU, his $\mathrm{PaO} 2 / \mathrm{FiO} 2$ ratio was 105 and he was classified as having moderate ARDS (Berlin criteria) with pulmonary haemorrhage. Due to his worsening oxygenation, he was started on a cisatracurium infusion that continued for 48 hours. He was ventilated using a standard low tidal volume approach (Volume $\mathrm{A} / \mathrm{C}$ mode with Tidal Volume (TV) of $315 \mathrm{~mL}$, PEEP of $14 \mathrm{cmH} 2 \mathrm{Oand}$ a flow rate of $35 \mathrm{~L} / \mathrm{min}$ ).

Despite fluid resuscitation, his systolic blood pressure remained less than $100 \mathrm{mmHg}$ and a noradrenaline infusion was started to maintain a mean arterial pressure of at least $65 \mathrm{mmHg}$. Noradrenaline dose reached a maximum of $13 \mu \mathrm{g} / \mathrm{min}$ for several hours on the first day decreasing to $<6 \mu \mathrm{g} / \mathrm{min}$ thereafter.

Due to the endemic nature of leptospirosis and his presentation with pulmonary haemorrhage, an atypical presentation of leptospirosis was considered. Although he had positive risk factors (swimming and rodent exposure), none of the typical features of leptospirosis were present (thrombocytopenia, jaundice or renal impairment). He was thus empirically started on ceftriaxone, levofloxacin and oseltamivir to cover possible leptospirosis and an atypical pneumonia.

On the second day of admission (day 7 of illness) leptospirosis IgM were reported as positive using a commercially available enzyme-linked immunosorbent assay. Subsequent dengue antibodies, rapid human immunodeficiency virus (HIV) test, urine, blood cultures and endotracheal aspirate were all negative.

The patient spent 7 days in the ICU on mechanical ventilation. Pulmonary haemorrhage persisted for the first four days during which he was heavily sedated on propofol and fentanyl infusions.

During his ICU stay, his platelet count, serum bilirubin and serum creatinine all remained normal. The patient was successfully extubated on day 7 onto a simple facemask with oxygen at $6 \mathrm{~L} / \mathrm{min}$. He spent a further 8 days in hospital complicated primarily by a severe myopathy.

\section{DISCUSSION}

Clinical data have shown that the frequency of pulmonary involvement in leptospirosis has increased over time and has become the primary presentation in certain areas. 2, 6,7 Anecdotally, we can attest to this predominance of pulmonary involvement in our setting. This is of particular importance to the Intensivist as most of these patients will require ICU care.

The incidence of pulmonary involvement in leptospirosis can range from $20-70 \%$ and has an estimated mortality rate in excess of $50 \% .^{2,8}$ Pulmonary involvement in leptospirosis can range from mild dyspnea and dry cough to severe pulmonary involvement (SPFL). Severe Pulmonary Form Leptospirosis (SPFL) includes haemorrhagic pneumonitis with massive pulmonary haemorrhage, isolated ARDS and ARDS with superimposed pulmonary haemorrhage. ${ }^{2,9,10}$ In a Brazilian study, leptospirosis presenting with massive pulmonary 
haemorrhage was associated with a $74 \%$ case-fatality rate. ${ }^{3}$ Previously, mortality from leptospirosis was mostly due to renal failure associated with Weil's disease, which is presently treatable in most critical care settings. ${ }^{6}$ The mortality rate from classic Weil's disease ranges from 5 $15 \% .^{1}$

The noteworthy feature in our patient was the absence of other associated signs of leptospirosis. In the Latin American region, jaundice was present in $93 \%$ and 95\% of leptospirosis cases in Brazil and Barbados respectively. ${ }^{1}$ In a short case series on anicteric leptospirosis and pulmonary haemorrhage by Assimakopoulos et al, all the patients had either associated thrombocytopenia or renal failure. ${ }^{11}$ In their patient population, Greece, the incidence of anicteric presentations of leptospirosis was $45 \%$.

Absence of any of these signs (jaundice, thrombocytopenia and renal failure) may delay the diagnosis and the institution of appropriate treatment. In non-endemic regions even with the presence of these associated signs, the diagnosis may still prove challenging. ${ }^{12} \mathrm{~A}$ high index of suspicion is thus warranted.

Fernando et al reported a case of ARDS secondary to leptospirosis without any of the more common clinical signs discussed above. ${ }^{9}$ Theirs was, however, a case of ARDS without any associated pulmonary haemorrhage, unlike our case. Although the exact pathophysiology of lung involvement in leptospirosis is currently unknown, two primary mechanisms are postulated. These are toxin mediated capillary vasculitis and a host mediated immune response. ${ }^{7}$ Histological findings in pulmonary leptospirosis show alveolar haemorrhage, congestion, alveolar oedema and hyaline membranes, features suggesting that both pulmonary haemorrhage and ARDS type changes can exist together. ${ }^{7}$ This may help to explain the poor oxygenation seen in these patients and the high levels of ventilatory support needed.

The diagnosis of leptospirosis remains difficult. The Leptospirosis Epidemiology Reference Group (LERG) defines a case of leptospirosis as one having a fourfold increase in microscopic agglutination titres (MAT) between acute and convalescent samples, a single MAT titre > 1:400 or detection of leptospira DNA using PCR. ${ }^{4}$ PCR based methods are the most accurate early in the disease ( $1^{\text {st }}$ week) with a reported sensitivity of $75 \% .{ }^{13}$ However, PCR is expensive and may not be available in all settings. MAT, the gold standard reference test has a sensitivity ranging from $23-41 \%$ in the first week of illness. ${ }^{4,}{ }^{13}$ Like PCR, MAT's availability is not universal. Rapid IgM screening tests are another option and become positive in the second week. These tests can be carried out by persons with limited laboratory training making the results more readily available for clinical use. Sensitivity varies depending on the test but averages $60 \%$ within the first 10 days. ${ }^{4} \mathrm{~A}$ combination of ELISA based IgM testing and clinical criteria was used in our case. The modified Faine's criteria proposed by the WHO for use in low resource regions combines both laboratory testing and clinical criteria and has a sensitivity of $>80 \% \cdot{ }^{5}$ A score of $>25$ suggests a presumptive diagnosis of leptospirosis, with our patient scoring 32 points.

Due to the low sensitivity and lack of availability of confirmatory leptospirosis testing, empirically starting treatment may be a feasible option especially when clinically suspected based on exposure (as in our case) and associated clinical features. ${ }^{14}$ In the ICU setting ceftriaxone is commonly used in the treatment of severe leptospirosis and may even prevent ICU admission in leptospirosis. ${ }^{15}$

Antibiotics are best given early on in the disease, which may be difficult to identify in atypical presentations. The importance of antibiotics in severe leptospirosis is debatable once pulmonary symptoms manifest and general supportive care may become of primary importance. ${ }^{2,16}$ ICU support in these patients may include hemodynamic support with vasopressors, renal replacement therapy and use of low tidal volume ventilation and positive end expiratory pressure (PEEP) in accordance with standard ARDS guidelines. ${ }^{17}$

Several specific therapies have been advocated to manage pulmonary complications of severe leptospirosis. Platelet transfusions are advocated to keep platelet counts $>50 \mathrm{x}$ $10^{3} / \mathrm{mm}^{3}$ and in keeping with the immune mediated mechanism of SPFL, pulsed methylprednisolone, cyclophosphamide, IV immunoglobulin and plasma exchange have all shown benefit. $2,18,19$ In one trial with 65 patients receiving ICU care, the addition of cyclophosphamide to pulse methylprednisolone showed a $66.7 \%$ survival rate compared to $9.4 \%$ in those who did not receive cyclophosphamide, however, most of their patients received only non-invasive ventilation. ${ }^{20}$ Further, high quality prospective studies are needed to determine the optimal therapy in patients with SPFL. 


\section{CONCLUSION}

Severe leptospirosis can present atypically with isolated ARDS and pulmonary haemorrhage without any other signs of organ involvement. It is important for clinicians to be aware of this mode of presentation as it is associated with a high mortality rate. A high index of suspicion is required especially where a potential exposure has occurred.

\section{Ethical approval statement: not required}

\section{Conflict of Interest: None declared}

\section{Informed consent statement: Obtained}

Author Contribution: KS wrote the main manuscript and NM wrote the case report

\section{REFERENCES}

1. Bharti AR, Nally JE, Ricaldi JN, Matthias MA, Diaz MM, Lovett MA, et al. Leptospirosis: a zoonotic disease of global importance. Lancet Infect Dis. 2003;3:757-71.

2. Marchiori E, Lourenco S, Setubal S, Zanetti G, Gasparetto TD, Hochhegger B. Clinical and imaging manifestations of hemorrhagic pulmonary leptospirosis: a state-of-the-art review. Lung. 2011;189:1-9.

3. Gouveia EL, Metcalfe J, de Carvalho AL, Aires TS, Villasboas-Bisneto JC, Queirroz A, et al. Leptospirosisassociated severe pulmonary hemorrhagic syndrome, Salvador, Brazil. Emerg Infect Dis. 2008;14:505-8.

4. Musso D, La Scola B. Laboratory diagnosis of leptospirosis: a challenge. $J$ Microbiol Immunol Infect. 2013;46:245-52.

5. Bandara K, Weerasekera MM, Gunasekara C, Ranasinghe N, Marasinghe C, Fernando N. Utility of modified Faine's criteria in diagnosis of leptospirosis. BMC Infect Dis. 2016;16:446.

6. Daher E, Zanetta DM, Cavalcante MB, Abdulkader RC. Risk factors for death and changing patterns in leptospirosis acute renal failure. Am J Trop Med Hyg. 1999;61:630-4.

7. Dolhnikoff M, Mauad T, Bethlem EP, Carvalho CR. Pathology and pathophysiology of pulmonary manifestations in leptospirosis. Braz J Infect Dis. 2007;11:142-8.

8. Gulati $S$, Gulati A. Pulmonary manifestations of leptospirosis. Lung India. 2012;29:347-53.
9. Fernando SM, Cardinal P, Brindley PG. Hypoxemic Respiratory Failure from Acute Respiratory Distress Syndrome Secondary to Leptospirosis. Case Rep Crit Care. 2017;2017:9062107.

10. Chauhan V, Mahesh DM, Panda P, Mokta J, Thakur S. Leptospirosis presenting as acute respiratory distress syndrome (ARDS) in sub-Himalayan region. $J$ AssoC Physicians India. 2010;58:390-1.

11. Assimakopoulos SF, Fligou F, Marangos M, Zotou A, Psilopanagioti A, Filos KS. Anicteric leptospirosisassociated severe pulmonary hemorrhagic syndrome: a case series study. Am J Med Sci. 2012;344:326-9.

12. Dall'Antonia M, Sluga G, Whitfield $S$, Teall A, Wilson $P$, Krahe D. Leptospirosis pulmonary haemorrhage: a diagnostic challenge. Emerg Med J. 2008;25:51-2.

13. Agampodi SB, Dahanayaka NJ, Nockler K, Anne MS, Vinetz JM. Redefining Gold Standard Testing for Diagnosing Leptospirosis: Further Evidence from a Well -Characterized, Flood-Related Outbreak in Sri Lanka. Am J Trop Med Hyg. 2016;95:531-6.

14. Suputtamongkol $Y$, Pongtavornpinyo $W$, Lubell $Y$, Suttinont C, Hoontrakul S, Phimda K, et al. Strategies for diagnosis and treatment of suspected leptospirosis: a cost-benefit analysis. PLoS Negl Trop Dis. 2010;4:e610.

15. De Francesco Daher E, Soares DS, de Menezes Fernandes ATB, Girão MMV, Sidrim PR, Pereira EDB, et al. Risk factors for intensive care unit admission in patients with severe leptospirosis: a comparative study according to patients' severity. BMC Infectious Diseases. 2016;16:40.

16. Charan J, Saxena D, Mulla S, Yadav P. Antibiotics for the treatment of leptospirosis: systematic review and meta-analysis of controlled trials. Int J Prev Med. 2013;4:501-10.

17. Papazian L, Aubron C, Brochard L, Chiche J-D, Combes A, Dreyfuss $D$, et al. Formal guidelines: management of acute respiratory distress syndrome. Annals of Intensive Care. 2019;9:69.

18. Shenoy W, Nagar VS, Chowdhury AA, Bhalgat PS, Juvale NI. Pulmonary leptospirosis: an excellent response to bolus methylprednisolone. Postgraduate medical journal. 2006;82:602-6.

19. Kularatne SA, Budagoda BD, de Alwis VK, Wickramasinghe WM, Bandara JM, Pathirage LP, et al. High efficacy of bolus methylprednisolone in severe 
leptospirosis: a descriptive study in Sri Lanka.

Postgraduate medical journal. 2011;87:13-7.

20. V Trivedi S, H Vasava A, C Patel T, Bhatia L.

Cyclophosphamide in pulmonary alveolar hemorrhage

due to leptospirosis. Indian journal of critical care

medicine : peer-reviewed, official publication of Indian

Society of Critical Care Medicine. 2009;13:79-84. 